\title{
Two Dimensional PEM Fuel Cell Modeling at Different Operation Voltages
}

\author{
Mohammad Ameri*, Pooria Oroojie \\ Energy Eng. Department, Power \& Water University of Technology, Tehran \\ * Tel: +98 2173932653, Fax: +98 2177311446,E-mail: ameri_m@yahoo.com
}

\begin{abstract}
This paper presents a comprehensive, consistent and systematic mathematical modeling for PEM fuel cells that can be used as the general formulation for the simulation and analysis of PEM fuel cells. As an illustration, the model is applied to an isothermal, steady state, two-dimensional PEM fuel cell at different operation voltages to investigate the fuel cells performance parameters such as the mass concentration, the velocity distribution of reactant, current density distribution, and polarization curve. The model includes the transport of gas in the gas flow channels, electrode backing and catalyst layers; the transport of water and hydronium in the polymer electrolyte of the catalyst and polymer electrolyte layers; and the transport of electrical current in the solid phase. Water and ion transport in the polymer electrolyte has been modeled using the generalized Stefan-Maxwell equations. Moreover, the reactant gas flow in the gas channel has been modeled by continuity and the steady state incompressible Navier-Stokes equations. All of the model equations are solved with finite element method using commercial software package COMSOL Multi physics. The results from PEM fuel cell modeling at different operation voltages are then compared with each other and finally according to the results, the strategy to improve fuel cell performance with the target of reducing cost is introduced.
\end{abstract}

Keywords: PEM fuel cell, Mathematical modeling, Finite element, COMSOL multi physic

\section{Introduction}

Fuel cells and hydrogen technology represent the most promising alternative pathway for automotive and stationary applications. The PEMFC offers low to zero emission from subwatt to megawatt power generation, for applications in transportation, industries and portable supplies units $[1,2]$. In this paper, we focus on the role of computational tools in order to verify some experimental results and demonstrate the better performance of PEMFC constructed by application of optimizations techniques. Experimental research and numerical simulation have been used in fuel cell design in order to improve the performance of fuel cells. The experimental data will be useful to validate the model.

The search for reliable computational models is a ch allenge because it involves several transport phenomena: multi-component, multi phase and multi-dimensional flow processes, electrochemical reactions, convective heat and mass transport in flow channels, diffusion of reactants through porous electrodes, transport of water through the membrane and transport of electrons through solid matrix. The Computational Fluid Dynamic (CFD) is a very useful tool to simulate hydrogen and oxygen gases flow channels configurations, reducing the costs of bipolar plates' production and optimizing mass transport [3-5].

The computational models are efficient in predicting the cell performance under a variety of design parameters. Fuel cell models can be classified into 1D, 2D and 3D according to dimensions. The accuracy of 1D model $[6,7]$ is sacrificed due to some assumptions made in order to simplify the problem to 1D. A 3D model simulates the reactant gas flow in the directions along the flow channel and perpendicular to the flow channel simultaneously, which results in more accurate results but requires longer computational time and larger computing capacity facility $[8,9]$. A 2D fuel cell model $[10,11]$ combines the benefits of $1 \mathrm{D}$ and 3D models and gains its popularity in PEM fuel cell modeling due to its higher computational efficiency compared to 3D models and better simulation accuracy compared to 1D model. A two-dimensional mathematical model of a PEM fuel cell can be conducted in two different modes: parallel or perpendicular to the gas flow direction in the gas channel 
while the other modeling dimension is across the membrane in both cases. Models conducted in the first mode (parallel to the gas flow direction) consider the influence of fluid behavior in the channel; while in the second mode (perpendicular to the gas flow direction) the interdigitated flow pattern can be easily investigated. The goal of the present work is to develop 2D isothermal, steady state PEM fuel cell models in perpendicular to the gas flow direction to investigate the performance of fuel cells such as the mass concentration and velocity distribution of reactants, polarization curve, output power density at different operation voltages so that one can examine the influence of operation voltage on those items.

\section{Modeling}

Fig. 1 schematically shows a 3D single PEMFC and its various components including the membrane, flow channels, gas diffusion layers and catalyst layers on both anode and cathode sides. To conduct a 2D simulation, there are two options to choose the modeling geometry: one is in $\mathrm{x}-\mathrm{z}$ plane as shown in Fig. 2(a), and the other is in $\mathrm{x}-\mathrm{y}$ plane as shown in Fig. 2(b). The geometry which is shown in Fig.2 (b) will be studied in this paper.

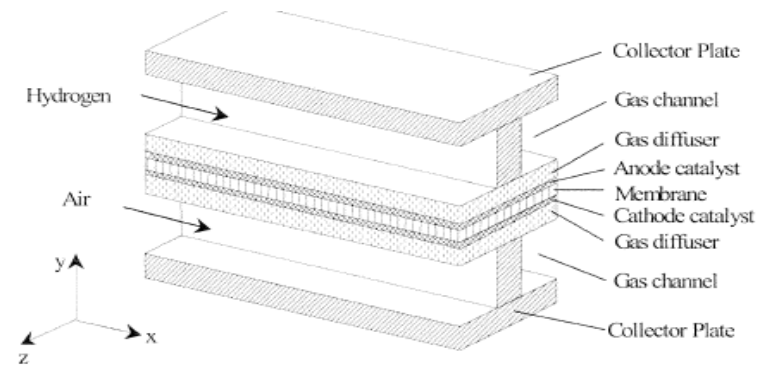

Fig.1. Three dimensional diagrams of a PEMFC and its various components.

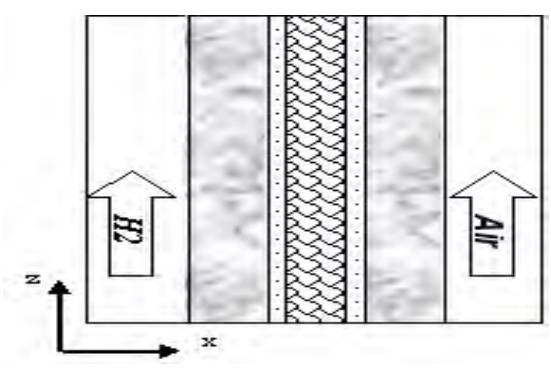

(a) $x$-z plane model geometry

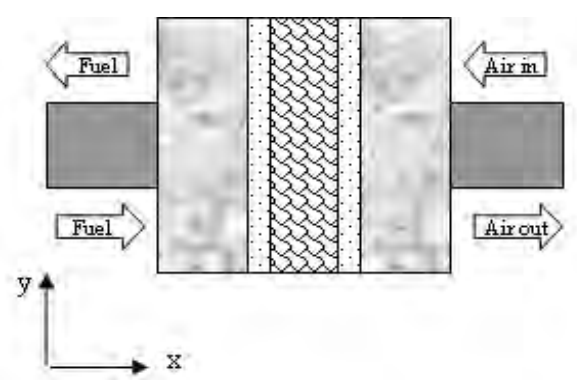

(b) $x$-y plane model geometry

Fig.2. Two-dimensional PEM fuel cell modeling geometry.

\subsection{Model Definition}

The modeled section of the fuel cell consists of three domains: an anode $\left(\Omega_{\mathrm{a}}\right)$, a proton exchange membrane $\left(\Omega_{\mathrm{m}}\right)$, and a cathode $\left(\Omega_{\mathrm{c}}\right)$ as indicated in Fig. 3(a). Each of the porous electrodes is in contact with an interdigitated gas distributor, which has an inlet channel $\left(\partial \Omega_{\mathrm{a} \text {, inlet }}\right)$, a current collector $\left(\partial \Omega_{\mathrm{a}, \mathrm{cc}}\right)$, and an outlet channel $\left(\partial \Omega_{\mathrm{a} \text {, outlet }}\right)$. The same notation is used for the cathode side. The model geometry is shown in Fig. 3(b). 


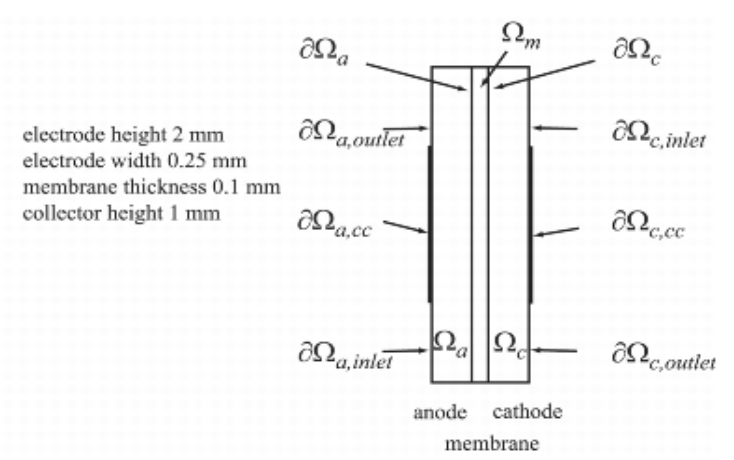

(a)Sub domain and boundary labels

Fig.3. Model geometry with sub-domain and boundary labels.

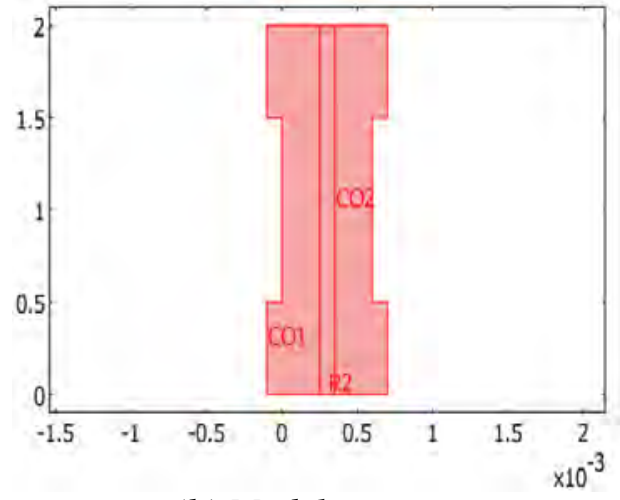

(b) Model geometry

\subsection{Governing Equations}

\subsubsection{Flow Channels}

Based on the model assumptions, the reactant gas flow in the gas channel is governed by the continuity equation to insure the mass conservation as well as the steady state incompressible Navier-Stokes equation to describe the momentum conservation of Newtonian fluids. The multi-component diffusion and convection in flow channels are described by the MaxwellStefan equation. It solves for the fluxes in terms of mass fraction. The general form of the Maxwell-Stefan equation is shown below.

$$
\begin{aligned}
& \frac{\partial}{\partial t} \rho \omega_{l}+\nabla \cdot\left[-\rho \omega_{l} \sum_{j=1}^{N} D_{i j}\left\{\frac{M}{M_{j}}\left(\nabla \omega_{j}+\omega_{j} \frac{\nabla M}{M}\right)+\right.\right. \\
& \left.\left.\left(x_{j}-\omega_{j}\right) \frac{\nabla P}{P}\right\}+\rho \omega_{l} u\right]=R_{i}
\end{aligned}
$$

Where $D_{i j}\left(\mathrm{~m}^{2} / \mathrm{s}\right)$ is the diffusion coefficient; $R_{i}\left(\mathrm{~kg} / \mathrm{m}^{3} . \mathrm{s}\right)$ is the reaction rate and it is zero in the flow channel; $\mathrm{x}$ is the mole fraction; $\omega$ is the mass fraction; $\mathrm{M}(\mathrm{kg} / \mathrm{mol})$ is the molecular mass; The subscript $i$ (or $j$ ) represents each species of hydrogen and water on the anode side, and oxygen, water, nitrogen on the cathode side. On the cathode side, the transport equations are solved for two species since the third species can always be obtained from the mass balance equation given as the following:

$$
w_{\mathrm{N}_{2}}=1-w_{\mathrm{O}_{2}}-w_{\mathrm{H}_{2} \mathrm{O}} ; w_{\mathrm{H}_{2} \mathrm{O}}=1-w_{\mathrm{H}_{2}}
$$

\subsubsection{Gas diffusion layers and catalyst layers}

Since gas diffusion layers (GDL) and catalyst layers are porous media, the velocity distribution is therefore formulated by Darcy's law and mass conservation equation.

$$
\begin{aligned}
& u=-\frac{\kappa}{\mu} \nabla p \\
& \nabla \cdot(\rho u)=S
\end{aligned}
$$

Where $\kappa\left(\mathrm{m}^{2}\right)$ is the permeability; and $\mu$ (Pa.s) is the dynamic viscosity. $\mathrm{S}$ is the source term, $\mathrm{kg} /\left(\mathrm{m}^{3} . \mathrm{s}\right)$. The continuity equation for the gas flow mixture describes the sum of all the involved gas species at each side. The source term, $\mathrm{S}$, accounts for the total consumption and 
production during the electrochemical reactions. In the catalyst layer, the reaction rate $R_{i}$ corresponding to each species is given as:

$$
R_{H_{2}}=-\frac{j_{a}}{2 F} M_{H 2} ; R_{O 2}=-\frac{\left|j_{c}\right|}{4 F} M_{O 2} ; R_{H_{2 O}}=\frac{\left|j_{c}\right|}{4 F} M_{H 2 O}
$$

\subsubsection{Current transport}

The continuity of current in a conducting material is described by:

$$
\nabla . i=\nabla . i_{s}+\nabla . i_{e}=0
$$

Protons travel through the ionic conductor (the membrane) to form an ionic current denoted by $i_{e}$ while electrons can only be transferred through the solid matrix of electrodes which results in an electronic current denoted by $i_{s}$. The potential equations for both solid and electrolyte phases are obtained by applying Ohmic's law to Eq. (6).

Electron transport: $\nabla \cdot\left(-\sigma_{s} \nabla \phi_{s}\right)=S_{s}$; Proton transport: $\nabla \cdot\left(-\sigma_{e} \nabla \phi_{e}\right)=S_{e}$

Where $\varphi$ is the phase potential, $\mathrm{V} ; \sigma(\mathrm{s} / \mathrm{m})$ is the effective electric conductivity; $\mathrm{S}\left(\mathrm{A} / \mathrm{m}^{3}\right)$ is the current source term; the subscript $\mathrm{s}$ denotes the property of a solid phase and e denotes the property of an electrolyte phase. The source terms in the electron and proton transport equations, result from the electrochemical reaction occurring in the catalyst layers of anode and cathode sides.

$$
\begin{aligned}
& \text { Anode Catalyst Layer: } S_{e}=j_{a} ; \quad S_{s}=-j_{a}, \\
& \text { Cathode Catalyst layer: } S_{e}=j_{c} ; S_{s}=-j_{a}
\end{aligned}
$$

Where $j_{a}$ and $j_{c}$ are the transfer current density corresponding to the electrochemical reaction at the anode and cathode catalyst layers, which is formulated by the agglomerate model. In the catalyst layers, the agglomerate is formed by the dispersed catalyst, and this zone is filled with electrolyte. Oxygen is dissolved into the electrolyte and reaches the catalyst site. The agglomerate model describes the transfer current density as following [12]:

Anode:

$$
\begin{gathered}
j_{a}=-\frac{6(1-\varepsilon) F D_{H}{ }^{a g g}}{\left(R^{a g g}\right)^{2}}\left(C_{H}^{a g g}-C_{H}{ }^{r e f} \exp \left(-\frac{2 F}{R T} \eta\right) .\right. \\
\left(1-\sqrt{\frac{j_{o, a^{s}}}{a F C_{H}^{r e f} D_{H}{ }^{a g g}}} R^{a g g} \operatorname{coth} \sqrt{\frac{j_{o, a^{s}}}{a F C_{H}^{r e f} D_{H}{ }^{a g g}}} R^{a g g}\right)
\end{gathered}
$$

Cathode:

$$
\begin{aligned}
& j_{c}=R \frac{12(1-\varepsilon) F D_{O}{ }^{a g g}}{\left(R^{a g g}\right)^{2}} C_{O}^{a g g} \cdot\left(1-\sqrt{\left.\frac{j_{o, c^{s}}\left(R^{a g g}\right)^{2}}{4 F C_{O}{ }^{r e f} D_{O}{ }^{a g g}} \exp \left(-\frac{0.5 F}{R T} \eta\right)\right) .}\right. \\
& \operatorname{coth} \sqrt{\frac{j_{o, c^{s}}\left(R^{a g g}\right)^{2}}{4 F C_{O}{ }^{r e f} D_{O}{ }^{a g g}} \exp \left(-\frac{0.5 F}{R T} \eta\right)}
\end{aligned}
$$


where $C^{a g g}\left(\mathrm{~mol} / \mathrm{m}^{3}\right)$ is the gas concentration at the surface of the agglomerates; $C_{\text {ref }}\left(\mathrm{mol} / \mathrm{m}^{3}\right)$ is the dissolved gas concentration at a reference state; $D^{a g g}\left(\mathrm{~m}^{2} / \mathrm{s}\right)$ is the diffusion coefficient of the dissolved gas inside the agglomerate; $R^{a g g}(\mathrm{~m})$ is the agglomerate radius; $j_{0}\left(\mathrm{~A} / \mathrm{m}^{2}\right)$ is the exchange current density; $\mathrm{s}\left(\mathrm{m}^{2} / \mathrm{m}^{3}\right)$ is the specific surface area; $\eta$ $(\mathrm{V})$ is the electrochemical over potential which is expressed by the potential difference between solid matrix and electrolyte and is defined as:

$$
\begin{aligned}
& \text { Anode side }: \eta=\phi_{s}-\phi_{e} ; \text { Cathode side: } \eta=\phi_{s}-\phi_{e}-U_{O C} \\
& \text { Open-Circuit Potential: } U_{O C}=1.23-0.9 \times 10^{-3}(\mathrm{~T}-298)
\end{aligned}
$$

The dissolved gas concentration at the surface of the agglomerates is corresponding to the molar fraction in the gas phase through Henry's Law:

$$
C^{a g g}=\frac{C_{g a s} P}{H}
$$

Where $\mathrm{H}\left(\right.$ pa.m $\left.\mathrm{m}^{3} / \mathrm{mol}\right)$ is the Henry's constant.

\subsection{Numerical Procedure}

COMSOL Multiphysics, which is a commercial solver based on the finite element technique, is used to solve the governing equations. The stationary nonlinear solver is used since the source terms of the current conservation equation make the problem non-linear. Furthermore, the convergence behavior of this non-linear solver is highly sensitive to the initial estimation of the solution. To accelerate the convergence, the following procedures are adopted. The Conductive Media DC module is firstly solved based on the initial setting. Secondly, Darcy's Law and Incompressible Navier-Stokes modules are solved together using the results from the previous calculation as initial conditions. After the previous two modules converge, all the coupled equations including Maxwell-Stefan Diffusion and Convection module are solved simultaneously until the convergence is obtained.

\section{Results and Discussion}

Using the aforementioned procedures, the $x-y$ geometry as described in Fig. 2(b) simulated in different voltages. The parameters values that used in this study are extracted from [13]. The $\mathrm{x}-\mathrm{y}$ model represents the PEM fuel cell with interdigitaed channels on the bipolar plate. Fig. 4 shows the current density curve obtained from the $x-y$ model at the anode active layer for a given operation voltage of 0.7 .

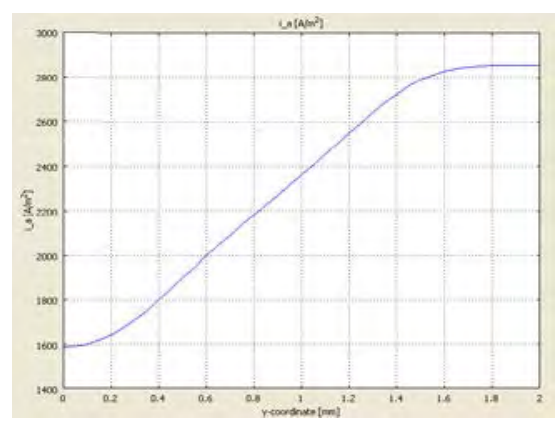

Fig.4. Current-density distribution at the anode active layer. 
The current density is uneven with the highest density in the cell's upper region. This means that the oxygen-reduction reaction rate in the cathode determines the current-density distribution. The maximum current density arises close to the air inlet. Figure 5 shows the current distribution in the PEM fuel cell for a given operation voltages of 0.7 and 0.6 . There are significant current spikes present at the corners of the current collectors and this trend will be more sensible at low voltages. The convective fluxes generally dominate mass transport in the cell. To study the convective effects, the velocity field is plotted in Fig.6. The flowvelocity magnitude attains its highest values at the current collector corners and it is more evident at lower operation voltages.

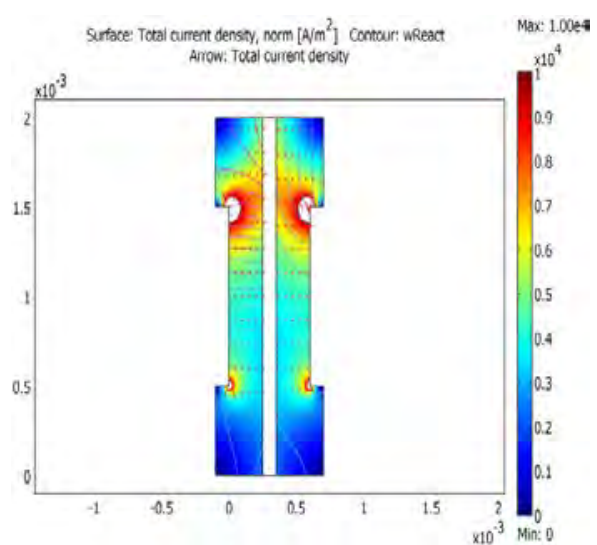

(a) 0.6 voltages

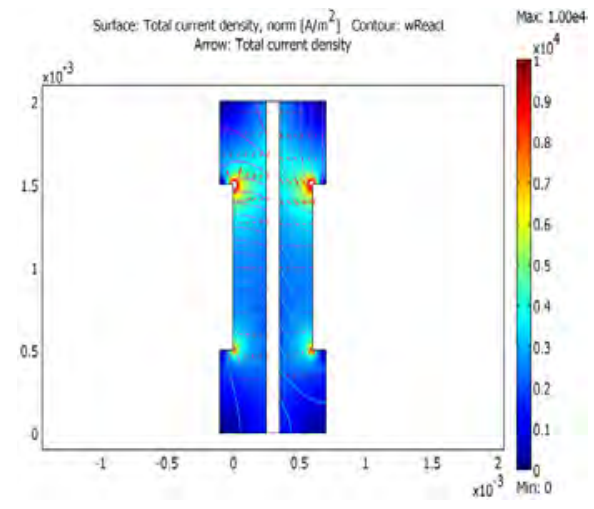

(b) 0.7 voltages

Fig.5. Current density (surface plot) and current vector field (arrow plot).

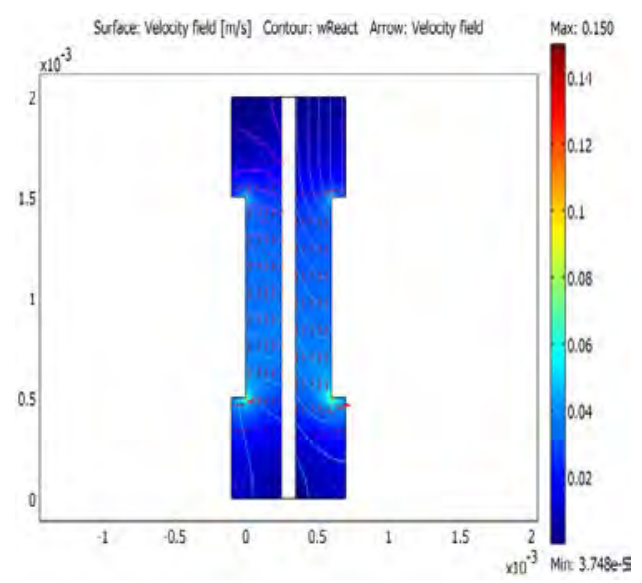

(a) 0.6 voltages

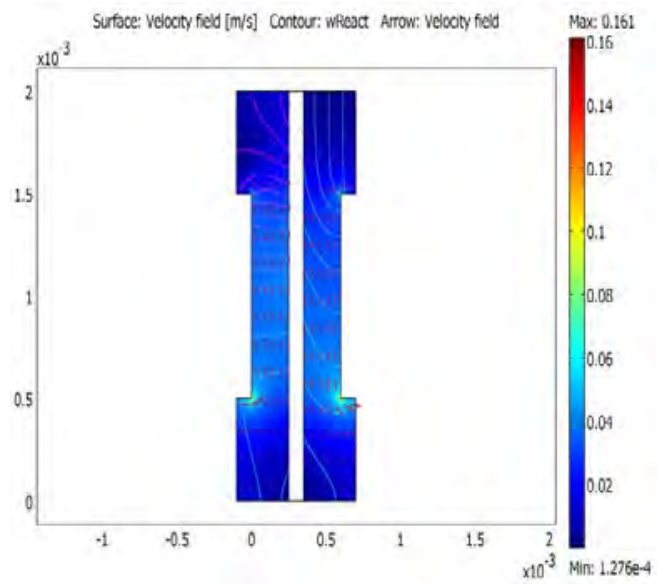

(b) 0.7 voltages

Fig.6. Gas velocity field in the anode and cathode compartments.

Figure 7 shows the reactant (oxygen and hydrogen) weight fractions in the cathode and anode gases. It is interesting to note that the hydrogen fraction increases as the anode gas flows from the inlet (at the bottom) to the outlet (at the top). This is the result of the electro osmotic drag of water through the membrane, which results in a higher flux than the consumption of hydrogen. This means that the resulting convective flux of anode gas towards the membrane causes the weight fraction of hydrogen to go up. In the cathode gas, there is an expected decrease in oxygen content along the flow direction and a small change in the oxygen flow gives a substantial change in cell polarization. Figure 8 depicts the water mass fraction in the anode and cathode gases as well as the diffusive flux of water in the anode. It is clear that water is transported through diffusion and convection to the membrane on the anode side. 


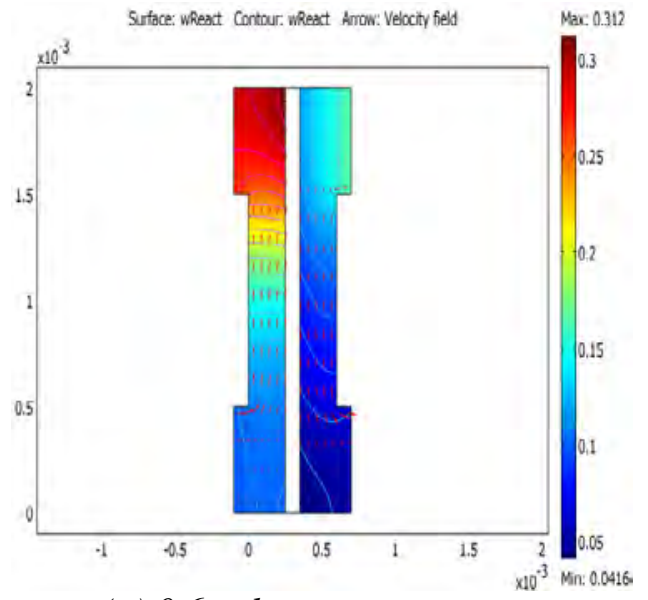

(a) 0.6 voltages

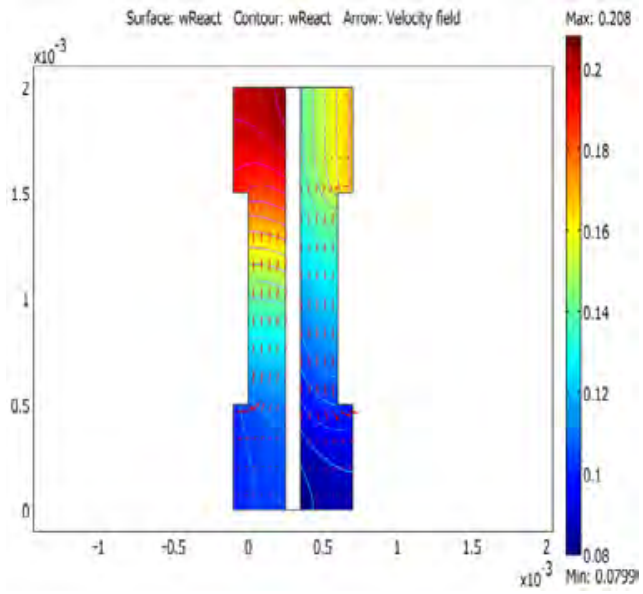

(b) 0.7 voltages

Fig.7.Normolized Reactant mass fractions, on the anode side (left) and cathode side (right).

The results show a minimum occurs at the upper corner of the membrane on the anode side that limit fuel cell performance. If the anode gas becomes too much dry, the membrane dries out, resulting in a decrease in ionic conductivity and failure of fuel cell. On the other hand, at the cathode side the water levels increase with the direction of flow and a local maximum in water current occurs at the lower corner of the membrane. This may also be critical since the water droplets can clog the pores and effectively hinder gas transport to the active layer.

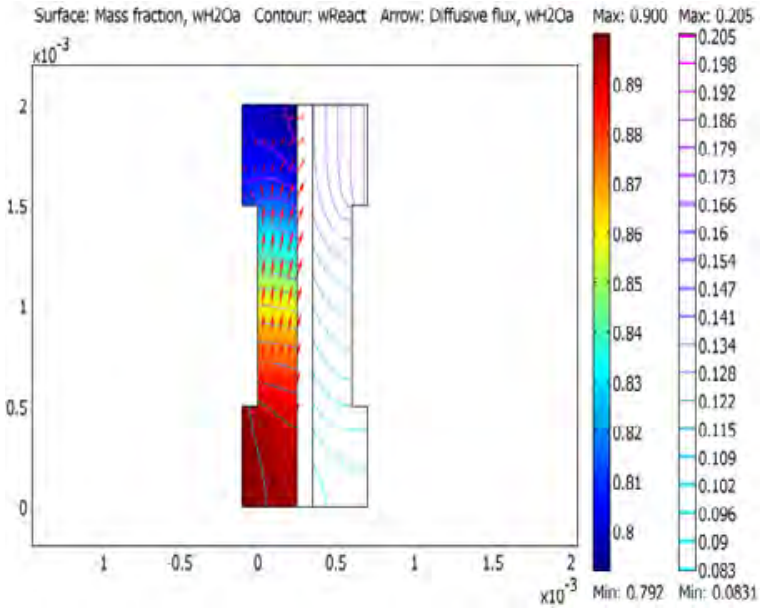

(a) 0.6 volt voltages

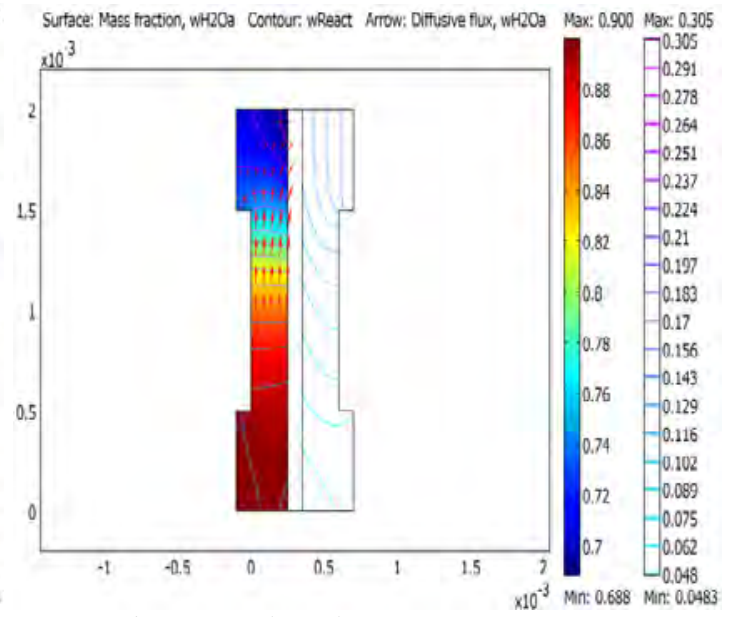

(b) 0.7 volt voltages

Fig.8. Water mass fraction in the anode (left, surface plot) and the cathode (right, contour plot). The arrows visualize the diffusive flux vector field on the anode side.

\section{Conclusion}

Two-dimensional, single-phase and isothermal models of PEM fuel cells have been developed at two different operation voltages. The model is able to investigate the transport phenomena and electrical potential distribution for the various PEM fuel cell components including the gas channels, gas diffusion layers, catalyst layers and membrane. The current density curve has been presented. The $\mathrm{x}-\mathrm{y}$ model used to study the fuel cell with interdigated channels design, shows that the fuel mass fraction decreases faster when the cell works at low voltage and high current density. The potential distribution indicates a major potential drop across the membrane. Moreover, a higher over potential on the cathode catalyst layer is noted. A minimum water mass fraction at upper corner of the membrane on anode side, reveals that 
this phenomenon is more sensible at low voltages. The flow velocity magnitude attains its highest value at the current collector corners and it is more intensified at low voltages.

\section{References}

[1] N. Djilali, Computational modeling of polymer electrolyte membrane (PEM) fuel cells, Challenges and Opportunities Energy, 2006, pp.1006-1016.

[2] E.F. Cunha, A.B. Andrade, E. Robalinho, M.L.M. Bejarano, E. Cekinski, M. Linardi, Modeling and simulation of PEM fuel cell's flow channels us ing CFD techniques, Proceeding of International Nuclear Atlantic Conference, 2007, pp.107-123.

[3] P.T. Nguyen, T. Berning, N. Djilali, Computational model of P EM fuel cell with serpentine gas flow channels, Journal of Power Sources, 130, 2004, pp.149-157.

[4] S. Karvonen, T. Rottinen, J. Saarinen, O. Himanen, Modeling of flow field in polymer Electrolyte membrane fuel cell, Journal of Power Sources, 161, 2006, pp. 876-884.

[5] G. Squadrito, O. Barbera, I. Gatto, G. Giacoppo, F. Urbani, E. Passalacqua, CFD analysis of the flow field scale-up influence on the electrodes performance in a PEFC, Journal of Power Sources, 152, 2005, pp. 67-74.

[6] T.E. Springer, T.A. Zawodzinske, S. Gatteadold, Polymer Electrolyte Fuel Cell Model, Journal of Electrochem Soc., 138, 2000, pp. 2334-2342.

[7] V. Gurau, F. Barbir, H. Liu, An Analytical Solution of a Half-Cell Model for PEM Fuel Cell, Journal of the Electrochemical Society, 147, 2000, pp. 2468-2477.

[8] T. Berning, D.M. Lu, N. Djilali, Three-dimensional computational analysis of transport phenomena in a PEM fuel cell, Journal of Power Sources, 106, 2004, pp. 284-294.

[9] K. Haraldsson, K. Wipke, Evaluating PEM fuel cell system models, Journal of Power Sources, 126, 2004, pp. 88-97.

[10] D.J. Nelson, M.R. Spakovsky, Single domain PEMFC model based on Siegel Siegel agglomerate catalyst geometry, Journal of Power Sources, 115, 2003, pp. 81-89.

[11] A. Biyikoglu, Review of proton exchange membrane fuel cell models, International Journal of Hydrogen Energy, 30, 2007, pp. 1181-1212.

[12]K. Broka, Characterization of the Components of the Proton Exchange Membrane Fuel Cell, Technical licentiate Thesis, Royal Institute of Technology, Stockholm, Sweden, 1995, pp. 93-107.

[13] H. Meng, C.Y. Wang, Model of Two Phase Flow and Flooding Dynamics in PEMFC, Journal of the Electrochemical Society, 152, 2005, pp.1733-1741. 